\title{
Implications of Color Models in Image Processing for Fire Detection
}

\author{
M. Senthil Vadivu \\ Assistant Professor, \\ Department of MCA, \\ Jyoti Nivas College, Bangalore
}

\author{
Vijayalakshmi M. N., PhD \\ Department of M.C.A, \\ R.V Engineering College, \\ Bangalore, India
}

\begin{abstract}
Detecting fire in the image is the recent research area in image rocessing.Fire causes damage to the environment, forest ecosystem, economy and devastation to life and property. Identifying fire in the early hours helps to decrease the damage and rescued from the risks. Traditional fire detecting methods are less capable to detect fire perfectly.Computer vision based methodologies has more reward on conventional algorithms in terms of accuracy and false alarms.Color models plays major role in recognizing fire pixels in the image.This paper portraits the implications of different color models employed in fire detection.
\end{abstract}

\section{Keywords}

Image Processing, Fire detection, Color Models

\section{INTRODUCTION}

Forest fire occurs often which is inevitable.It is severe fact that will cause huge loss to the life, environment,ecology. Along with these effects , it also spoils the soil culture, plants, organization of the forest.The early detection of fire will help to reduce these problems and to save the nature[1][13] .

The main aspect of real target detection is detect fire in dangerous situation and in complicated scene. This will be a challenging tasks when there is less difference between background and the foreground part.

Computer vision based approaches are more promising for detecting the fire in the early stage with accuracy.There are numereous existing approaches in the literature which detects fire in different ways.Some of the methods are based on edges of fire, different color proposition of fire.The research says color models play vital role in detecting fire powerfully than other the methods[4][6][8].A fire pixel can be determined by the color pixels which lie in the predermined range of intensity values.Usually there are pixels which resembles the fire color pixels like orange, Red color dress, apple, sunset, sunshine etc.There is a need to discriminate these pixels into fire and non fire pixels. Getting a glimpse of the color models will help to choose among them to propose a new method. This paper focuses on inference of different color models in identifying the fire[9]

Numerous colour models are existing in image processing like RGB, HSV, HSI, YUV, YCbCr, CIE L*a*b*[6]. RGB color model is quite easy to implement along with linearity.It is device dependent and all the three colors are perceptually non-uniform.If there is a high change in illumination then this

color model would not be a promising appraoch to aquire better results.HSV color models deals with Hue, Saturation and Value of an image. Hue gives the high illumination data which supports to detect fire.This is better than RGB model in terms of recall an precision.The main disadvanateg is tinting ie desaturated colors having increased total intensity. CIE $\mathrm{L}^{*} \mathrm{a} * \mathrm{~b} *$ model is more perceptually linear than any other color models.Moreover it is device independent . CMYK color model is device dependent but non linear with visual perception .But conversion between RGB and CMYK will be complicated. Ycbcr models refers $\mathrm{Y}$ as luminance, $\mathrm{Cb}$ as chrominance blue, $\mathrm{Cr}$ as chrominance red .It is more supportive in image and video compression standards. But it is perceptive and device dependent.Each models has played better role in determining fire in the images and videos[1][3][10].

This paper is organised into as follows : Section II discusses the existing approaches in the literature survey, section III gives general description of various color models helps in detecting fire. Section IV concludes the paper

\section{RELATED WORK}

There are several methods in the literatures developed for fire flame detection from video images. Most of the current methods use motion and color information to detect the flame.Toreyin et al[1] identified fire regions using color,motion and fire frequency in videos. In his work he adapated Hidden Markov Model based computed the difference between current frame and previous frame to find the moving pixel regions. Yusuf Hakan et al [2] has proposed a method to detect fire by uisng color,spatial and temporal information.This approach uses covariance based features. The extracted features are classified using SVM Classifier. Grech-Cini [3] used a rule-first Bayesian-next analysis method to detect fire and smoke with image change,correlation,variance. $\mathrm{Xu}$, Zhengguang et al [4] detected fire and smoke by using the features like moving target including growth, disorder,frequent flicker in boundaries, self similarity and loal wavelet energy. Zhai, Wenpeng et al [5] proposed a method to identify fire in the early stage to reduce the false alarm by using dynamic features and color.

\section{COLOR MODELS FOR FIRE DETECTION}

Each color space has its own pros and cons.Adopting a particular model will be dependent on the problem.This section gives some existing approaches with different color models which helps in detecting fire in the images.It would be a moviating factor to choose different color spaces

\subsection{YCbCr Color Space Model}

$\mathrm{Y}$ is the luma component and $\mathrm{Cb}$ and $\mathrm{Cr}$ are the blue difference and red-difference chroma components. $Y$ is refered as luminance. It is not an absolute color space. Y which is luminance,meaning that light intensity is nonlinearly 
encoded based on gamma corrected RGB primaries[12]. $\mathrm{YCbCr}$ is not an complete color space. $\mathrm{YCbCr}$ which refers to the luminance component $\mathrm{Y}, \mathrm{Cb}$ refers to the blue color component, and $\mathrm{Cr}$ refers to the red color component. The human eyes are more receptive for the component $\mathrm{Y}, \mathrm{YCbCr}$ is conventional only if standard $\mathrm{RGB}$ primary chromaticties are used.

Most of the images will be in R G B color space.There is need to convert the RGB image into $\mathrm{YCbCr}$. Because the flame color of those pictures are spread more uniformly in $\mathrm{YCbCr}$ color space[13]

The conversion formula from $\mathrm{RGB}$ color space to $\mathrm{YCbCr}$ is as formula one follow.

\section{$\mathrm{RGB}->\mathrm{YCbCr}$}

$\mathrm{Cr}=0.50 .5 * \mathrm{R}-0.418688 * \mathrm{G}-0.081312 * \mathrm{~B}$;

$\mathrm{Cb}=0.5-0.168736 * \mathrm{R}-0.331264 * \mathrm{G} 0.5 * \mathrm{~B}$;

$\mathrm{Y}=0.299 * \mathrm{R} 0.587 * \mathrm{G} 0.114 * \mathrm{~B}$

Among them, $\mathrm{Y}$ is the luminance component, $\mathrm{Cr}$ reflects the difference between the red component and the illumination value of the input RGB signal, and $\mathrm{Cb}$ reflects the difference between the blue component and the illumination value of the input RGB signal, the value ranges for those components is between 0 and 255 .

Rui Chen[8] et al has proposed a method to detect fire pixels by using $\mathrm{YbCbCr}$ and $\mathrm{K}$-means algorithm The allocation of $\mathrm{Cb}$ and $\mathrm{Cr}$ most likely close to normal distribution, and in the $(\mathrm{Cb}, \mathrm{Cr})$ color space, the distribution also mostly in line with bivariate normal distribution. The function of bivariate normal distribution is as formula four follow

$F(x, y)=\frac{1}{2 \pi \sigma_{x} \sigma_{y}} \exp \left[\frac{1}{2}\left[\frac{\left(x-\mu_{x}\right)^{2}}{\sigma_{x}{ }^{2}}+2 \frac{\left(x-\mu_{x}\right)\left(y-\mu_{y}\right)}{\sigma_{x} \sigma_{y}}+\frac{\left(y-\mu_{y}\right)^{2}}{\sigma_{y}{ }^{2}}\right.\right.$

Among them, $\mu_{x}$ is the expectation of $x, \sigma_{x}$ is the standard deviation of $x, \mu_{y}$ is the expectation of $y, \sigma_{y}$ is the standard deviation of $y$.

By using the simple variance and standard deviation of $\mathrm{Cb}$ and $\mathrm{Cr}$ replace mean and standard deviation. Hence the transformed formula is as

$F(c r, c b)=\frac{1}{2 \pi S_{C r} S_{C b}} \exp \left[\frac{1}{2}\left[\frac{(C r-\overline{C r})^{2}}{S_{C r}}+2 \frac{(C r-\overline{C r})(C b-\overline{C b})}{S_{C r} S_{C b}}+\frac{(C b-\overline{C b})^{2}}{S_{c b}}\right.\right.$

Here $\overline{C r}, \overline{C b}, S_{C r}, S_{C b}$ are simple variance and simple deviation and the calculation formula is

$\overline{\mathrm{cr}}=\frac{1}{N} \sum_{i=1}^{N} C_{\mathrm{r}}$

$\mathrm{SCr}^{2}=\frac{1}{N} \sum_{i=1}^{N}\left(C r_{\mathrm{i}}-\overline{\mathrm{Cr}}\right)^{2}$

The probability of flame pixel s can be determined by finding the value of $\mathrm{Cr}$ and $\mathrm{Cb}$. The respective flame pixel can be identified by using a threshold T If $f(\mathrm{Cr}, \mathrm{Cb})>T$. Threshold $\mathrm{T}$ can be chosen by finding the probability of the existence of the flame pixel.Equation (1)-(3) shows the formulas to determine

The decision rule of the flame pixel as follow. else not fire pixel;
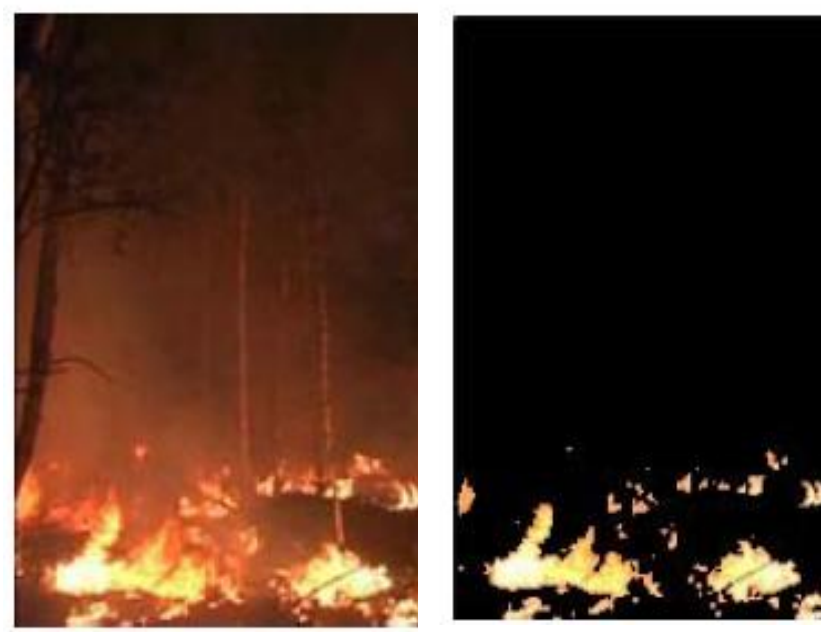

Figure 1. (a)Original Image b)After segmentation

$\mathrm{K}$-means algorithm[8] has attracted towards many areas ranging from segmentation,computer vision,geostatistics, astronomy, agriculture and so on.The reason behind to utilize this algorithm is easy to implement and can be applied on big data sets.Moreover it follows iterative approach,at each iteration, new centroid will be selected .In the above method the fire pixels are classified by using K-means algorithm.Figure 1 shows the original image and the proposed results

\subsection{RGB Color Model}

Sanqi Li et al[9] has proposed a fire detection model by using RGB color model. In this color model ,the color pixels can be extracted into Red, Green and Yellow components .Pixel at each location can be represented as $\mathrm{R}(\mathrm{x}, \mathrm{y}), \mathrm{G}(\mathrm{x}, \mathrm{y})$ and $\mathrm{B}(\mathrm{x}, \mathrm{y})$. The rules to define the fire pixels are

Rule1: R > RTH

Rule2: $\mathrm{R} \geq \mathrm{G} \geq \mathrm{B}$.

\section{Rule 3: $\mathrm{S} \geq(255-\mathrm{R}) * \mathrm{STH} / \mathrm{RTH}$}

The fisrt rule says that Red component in each pixels which are greater than red threshold value will be extracted from the image.Second rule concentrates on the Red color than intensities of all other colors. Since $\mathrm{R}$ is a major color in the fire with more brightnes[7][12].Sometimes the background with high illumination may influence the saturation of flames.To reduce the false alarms, the extract the flame intensity greater than a specified threshold. RTH value can be choosed experimentally.In this experiment 180 is chosen as RTH

if $f(C r(x, y), C b(x, y))>T$ then fire pixel 

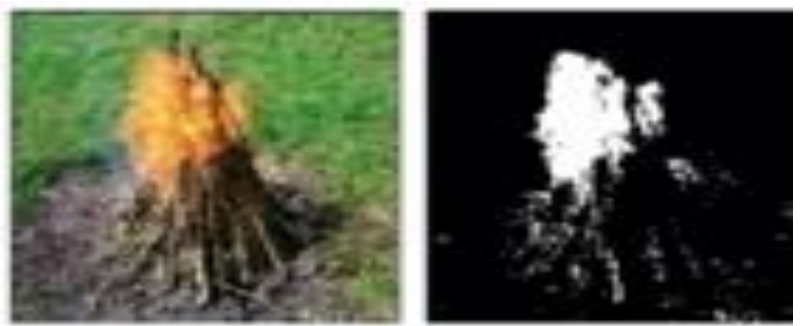

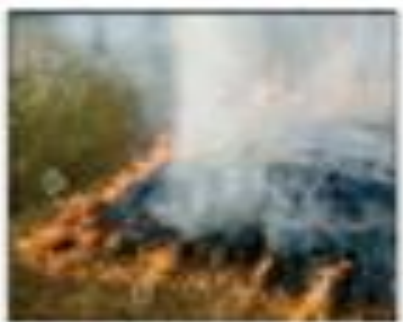

Figure 2. a) Original Images

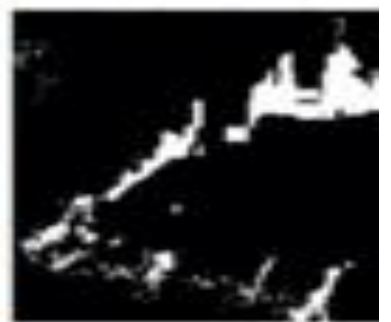

b)After Segmentation
Figure shows the original image and segmented fire region.

But RGB color model has its own limitations.There is a high correlation among all the three colors. Change in one color component may adversley affect the other color.It is device dependent.

\subsection{CIE Lab Color Model}

This color model is recognized by International Commission on Illumination(CIE) in 1976.It illustates all the colors which are visible to the human eye.The main advantage of implementing this model is device independent.It is known as uniform color space. The lightness or Luminance or Brightness $\mathrm{L}^{*}$, stand for the darkest black at $\mathrm{L}^{*}=0$, and the brightest white at $\mathrm{L}^{*}=100$. The $\mathrm{a}^{*}$ and $\mathrm{b}^{*}$ color channels, will match up to neutral gray values when $a^{*}=0$ and $b^{*}=0$. In the $\mathrm{a}^{*}$ axis the red color provides positive values and green with negative values [16]. Similarly in $b^{*}$ axis, blue colour represents negative values and yellow as positive values.Conversion from RGB to CIE Lab color space and the non-linear relations of $\mathrm{L}^{*}, \mathrm{a}^{*}$ and $\mathrm{b}^{*}$ are shown in equation (4)

$\left[\begin{array}{l}X \\ Y \\ Z\end{array}\right]=\left[\begin{array}{lll}0.412453 & 0.357580 & 0.180423 \\ 0.212671 & 0.715160 & 0.072169 \\ 0.019334 & 0.119193 & 0.950227\end{array}\right] \times\left[\begin{array}{l}R \\ G \\ B\end{array}\right]$

$\mathrm{L}^{*}= \begin{cases}116^{*}\left(y / y_{n}\right)^{1 / 3}-16, & \text { if }\left(y / y_{n}\right)>0.008 \\ 903.3 *\left(y / y_{n}\right), & \text { Otherwise }\end{cases}$

$\mathrm{a}^{*}=500 *\left(f\left(x / x_{n}\right)-f\left(y / y_{n}\right)\right.$,

$\mathbf{b}^{*}=200 *\left(f\left(y / y_{n}\right)-f\left(z / z_{n}\right)\right)$,

$\mathrm{f}(\mathrm{t})= \begin{cases}t^{1 / 3}, & \text { ift }>0.008856 \\ 7.787 * t+16 / 116, & \text { Otherwise }\end{cases}$

Where $\mathrm{Xn}, \mathrm{Yn}$, and $\mathrm{Zn}$ are the tri-stimulus values of the reference colour white.

It is assumed that the fire in an image has the brightest image region and is near to red color.

T. Celik et al[7] has defined a methodology by using RGB color model and statistical data .They have defined some set of fllowing rules to determine fire pixels.

$$
\begin{aligned}
& R 1(x, y)=\left\{\begin{array}{l}
1, \text { if } L *(x, y) \geq L * m \\
0 \quad \text { Ot } \square \text { rwise }
\end{array}\right. \\
& \mathrm{R} 2(x, y)=\left\{\begin{array}{c}
1, \text { if } a *(x, y) \geq a * m \\
0, \text { ot } \square \text { erwise }
\end{array}\right. \\
& \mathrm{R} 3(x, y)=\left\{\begin{array}{c}
1, \text { if } b *(x, y) \geq b * m \\
0, \text { ot } \square \text { erwise }
\end{array}\right. \\
& \mathrm{R} 4(x, y)=\left\{\begin{array}{c}
1, i f b *(x, y) \geq a *(x, y) \\
0, \text { ot } \square \text { erwise }
\end{array}\right. \\
& \mathrm{F}(x, y)=\left\{\begin{array}{c}
1, \text { if } \sum R i(x, y)=4 \\
0, \text { ot } \square \text { erwise }
\end{array}\right.
\end{aligned}
$$

Here R1,R2,R3,R4 are binary images which displays the presence of fire pixels in the image.Equation (5)-(9) gives the expressions .Finally the pixels which are not satisfied under these rules will be subtracted from the pixel.Later the selected fire colored pixels will be passed to a classification algorithm to classify the pixel is fire pixel or not[6][13]

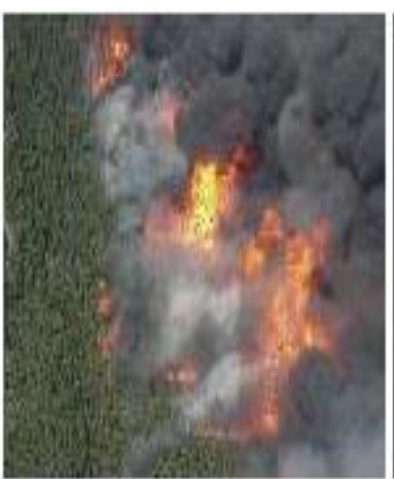

Figure 3.a) Original Image

The above Figure shows the segmented fire pixels in the image

\section{CONCLUSION}

This paper emphasized on how different color models are used to the presence of fire pixels in the image.Each model has its own advantages and disadvantages. This article will give an idea about the influence of color models to predict the fire in the image.Numerous methods are existing in the literature.But methods are still lacking in accuracy and providing false alarms. There is a future scope to improve the efficiency of the existing models in a better way by considering other features like smoke, texture, dynamic properties of fire,edges of fire pixels. These combined features can help to increase the proportion of true positives of the fire subsistence in the image

\section{REFERENCES}

[1] B. U. Toreyin, A. E. Centin, "Online detection of fire in video," IEEE. Conf. on Computer Vision and Pattern Recognition, pp. 1 - 5, June 2007.

[2] Yusuf Hakan Habiboglu. Osman Günay. Cetin, A.E. "Covariance matrix-based fire and flame detection method in video". Machine Vision and Applications. DOI 10.1007/s00138-011-0369-12011.

[3] H. J. Grech-Cini, "Smoke Detection", US Patent No. US6844818B2.. 
[4] $\mathrm{Xu}$, Zhengguang, $\mathrm{Xu}$, Jialin. "Automatic fire smoke detection based on image visual features". ProceedingsCIS Workshops 2007, International Conference on Computational Intelligence and Security Workshops, 2007, pp. 316-319.

[5] Zhai, Wenpeng, Wu, Aiguo; Du, Chunyan. "Dynamic features and color feature based fire smoke detection". Proceedings of the 29th Chinese Control Conference, CCC'10, 2010, pp. 3052-3055

[6] P. V. K. Borges, E. Izquierdo, "A probabilistic approach for visionbased fire detection in videos," IEEE. Trans. On Circuits and Systems for Video Technology, vol. 20, pp. 721 - 731, may 2010.

[7] T. Celik, H. Demirel, H. Ozkaramanli, M. Uyguroglu, "Fire detection using statistical color model in video sequences", Journal of Visual Communication and Image Representation (2007) 176-185.

[8] Rui Chen, Yuanyuan Luo, Mohanmad Reza Alsharif," Forest Fire Detection Algorithm Based on Digital Image",Journal of Software , Vol. 8, No. 8, August 2013,PP-1897-1905
[9] Sanqi Li,Wenbin $\mathrm{Li}^{*}$,Jiangming kan, yutan wang, " An Image Segmentation Approach of Forest Fire Area based on Aerial Image ", Journal of Theoretical and Applied Information Technology 15th December 2012. Vol. 46 No.1,PP 207-211.

[10] P.T. Bharti, Dr. P. Subhashini, Wseas- "Optimization of image processing techniques using Neural Networks:A review", Transactions on Information Science and Applications ISSN: 1790-0832 Issue 8, Volume 8, August 2011

[11] Mukesh Kumar, Pedro gomes and RohiniSaxena- "A Vision Based Approach To Fire Detectio"-,International Journal of Advanced Robotic Systems, Received 24 Apr 2014; Accepted 18 Jun 2014.

[12] Gaurav Yadav- "Optimized Flame Detection using image processing based techniques", Indian Journal of Computer Science and Engineering (IJCSE), ISSN: 0976-5166 Vol. 3 No. 2 Apr-May 2012.

[13] S.P. Kale et al Int- "Novel Technique for Fire detection". Journal of Engineering Research and Applications, ISSN: 2248-9622, Vol. 4, Issue 1(Version 3), January 2014, pp.203-205. 